\title{
Incidence of postoperative wound infections after open tendo Achilles repairs
}

\author{
Mohd Mizan Marican ${ }^{1}$, MBBS, FRCS, Stephanie Man Chung Fook-Chong ${ }^{2}$, MSc, CStat, Inderjeet Singh Rikhraj'1 ${ }^{1}$ MBBS, FRCS
}

\begin{abstract}
INTRODUCTION Tendo Achilles (TA), which is the confluence of the gastrocnemius and soleus muscles, is one of the most commonly injured tendons. The surgical repair of TA ruptures is associated with a significant risk of infection. This study examined several factors (i.e. gender, age, body mass index, history of diabetes mellitus, steroid use, acute or chronic TA injuries, type of surgical incision and type of sutures used) that may be associated with postoperative wound infection after open TA repair.

METHODS This was a retrospective study involving 60 patients who underwent open TA repair over an 18-month period. Patients who had prior TA surgery or open TA injuries, or who needed soft tissues flaps were excluded.

RESULTS Among the patients, 7 (11.7\%) developed superficial wound infections that were successfully treated with oral antibiotics, while $3(5.0 \%)$ developed deep wound infections that required at least one debridement procedure. No significant association was found between the risk of postoperative wound infection and gender, age, the presence of diabetes mellitus, acute or chronic ruptures, site of surgical incision and type of deep or superficial sutures used.

CONCLUSION While diabetes mellitus and age did not appear to be associated with postoperative wound infections after open TA repair, obese patients were found to be two times more likely to develop a wound infection than normal-weight patients. The incidence of superficial wound infections in this study was similar to previously published results ( $11.7 \%$ vs. $8.2 \%-14.6 \%)$, but the incidence of deep infections was higher $(5 \%$ vs. $1 \%-2 \%)$.
\end{abstract}

Keywords: Achilles tendon rupture, risk factors, wound infection

\section{INTRODUCTION}

Tendo Achilles (TA) is the confluence of the gastrocnemius and soleus muscles. Although it is the strongest tendon in the body, it is one of the most commonly injured tendons in the adult population, especially in men in their third and fourth decades of life.(1) TA ruptures usually occur during running, jumping, sprinting and other activities where explosive plyometric contractions or rapid eccentric loading occur. ${ }^{(2)}$ It is widely believed that TA ruptures occur in previously abnormal tendons. ${ }^{(2,3)}$ Abnormal tendons can be found in persons with tendon degeneration, mechanical abnormalities of the foot, or medical conditions such as diabetes mellitus or arterial occlusions; persons under the adverse influence of medications such as steroids and Ciprofloxacin antibiotics; and persons exposed to hypothermic conditions or active in endurance sports. $^{(4-6)}$

Surgical treatment for TA ruptures leads to good recovery and low re-rupture rates; however, it is also associated with complications. ${ }^{(3,4)}$ Major complications include re-ruptures, an equinus position of the foot, excessive lengthening of the tendon rendering the ankle weak, deep infections, skin necrosis, deep vein thrombosis (DVT) and pulmonary emboli (PE). Minor complications include superficial infections, delayed wound healing, haematomas, scar sensitivity and suture granulomas. ${ }^{(4)}$ Surgery for TA ruptures is associated with general skin healing complication rates of about $8.2 \%-14.6 \%$ and deep wound infection rates of $1 \%-2 \%{ }^{(4,5,7-9)}$ Previous studies have shown that outcomes are satisfactory after simple re-ruptures without infection, but are distinctively poor if the re-rupture involves deep infections. ${ }^{(8,9)}$

In the present study, we aimed to establish the incidence of wound infection among patients who underwent open surgical repair of TA ruptures in our institution. We also assessed whether there was any correlation between postoperative wound infections and patient demographics, surgical technique used or antibiotics used.

\section{METHODS}

Records from the Department of Orthopaedic Surgery, Singapore General Hospital, were reviewed to identify all patients who underwent open primary repair for midsubstance TA ruptures over an 18-month period (between January 2008 and June 2010). The diagnosis of TA rupture was made clinically, based on physical examination, or ultrasonography if the diagnosis was uncertain. The records were retrospectively reviewed and data was extracted, such as patient age, body mass index (BMI), smoking habits, history of diabetes mellitus, use of corticosteroids and type of TA rupture at the time of surgery (acute [i.e. less than six weeks old] or chronic). We also recorded surgical details such as the surgical approach used and the use of pre- and postoperative antibiotics. Patients who met the following criteria were excluded from this study: (a) a history of previous TA surgery on the same leg; (b) a history of open and avulsion TA injuries; (c) had injuries that required soft tissue flaps to augment the TA repair; and (d) had follow-up within the preceding two years. The patients who were included in the study had agreed to surgery

${ }_{1}^{1}$ Department of Orthopaedic Surgery, ${ }^{2}$ Division of Research, Singapore General Hospital, Singapore

Correspondence: Dr Mohd Mizan Marican, Associate Consultant, Department of Orthopaedic Surgery, Singapore General Hospital, 20 College Road, Academia Level 4, Singapore 169856. mohd.mizan.marican@singhealth.com.sg 
after being individually counselled by their respective surgeons on the risks and benefits of both the conservative and surgical routes of management for their injury. The follow-up period for this study was two years. Patients who were not seen in the clinic after several months and were not willing to return to the clinic for follow-up after two years were contacted to enquire about the presence of infections over their surgical site.

In the immediate postoperative period, the patients' ankles were fitted with a front slab and plantar-flexed to about $30^{\circ}$ to $40^{\circ}$. The patients were kept on a strict non-weight-bearing status (crutches were to be used to move about) and they were encouraged to keep their foot elevated to prevent excessive swelling. About two weeks after surgery, the patients were reviewed in the clinic; their wounds were inspected and their stitches removed. The patients were then given a plaster cast and crutches for about 2-3 more weeks. The clinic's physiotherapists followed up on all the patients and taught them active range of movement exercises, such as isometric ankle inversion and eversion, and dorsi and plantar flexion of their ankles. Full weight bearing, calf stretching, and static balance and ankle strengthening exercises were initiated about 4-6 weeks after surgery. These rehabilitation exercises were introduced progressively, depending on the patients' pain thresholds (visual analogue score $<5$ ), motivation and flexibility. For example, patients who were actively pursuing sporting hobbies may have aimed to achieve greater targets with their physiotherapists in an attempt to return to sports quickly, while elderly patients and those with low physical demands may have chosen to continue physiotherapy until they could walk independently and perform their daily activities without any restrictions.

The data in the present study was analysed using SPSS Statistics version 17.0 (SPSS Inc, Chicago, IL, USA). The summary statistics for continuous variables were expressed as mean \pm standard deviation. We attempted to calculate the statistical significance between variables using the generalised estimating equation (GEE) model. However, as the GEE failed to converge, Fisher's exact test was used instead. A p-value $<0.05$ indicated statistical significance. The primary outcome measured was surgical wound site infection.

\section{RESULTS}

A total of 76 patients underwent open TA repair over the 18 months. Of these 76 patients, 60 (33 male, 27 female) were included in the present study; 16 patients were excluded for a variety of reasons: nine were not contactable, four had undergone TA repair and flap surgery, and three had undergone revision TA surgery.

The surgeries of the 60 patients included in the present study were performed by a total of ten different surgeons. They were conducted with the patients in the prone position, with the tourniquet applied. After surgery, all 60 patients had their ankles immobilised in slight plantar flexion with a front slab. The patients were taught how to use crutches before their discharge. None of the patients were on long-term oral steroids or topical corticosteroids.
Table I. Incidence of postoperative wound infections after open tendo Achilles repair, according to the patient demographics of the study population $(n=60)$.

\begin{tabular}{|c|c|c|c|}
\hline \multirow{2}{*}{$\begin{array}{l}\text { Demographic/ } \\
\text { surgical factor }\end{array}$} & \multicolumn{2}{|c|}{ No. (\%) } & \multirow[t]{2}{*}{ p-value } \\
\hline & $\begin{array}{c}\text { With } \\
\text { infection } \\
(n=10)\end{array}$ & $\begin{array}{c}\text { Without } \\
\text { infection } \\
(n=50)\end{array}$ & \\
\hline Gender & & & 0.497 \\
\hline Male $(n=33)$ & $5(15.2)$ & $28(84.8)$ & \\
\hline Female $(n=27)$ & $5(18.5)$ & $22(81.5)$ & \\
\hline Age group (yr) & & & 0.472 \\
\hline $20-29(n=7)$ & $0(0)$ & $7(100.0)$ & \\
\hline $30-39(n=13)$ & $3(23.1)$ & $10(76.9)$ & \\
\hline $40-49(n=12)$ & $2(16.7)$ & $10(83.3)$ & \\
\hline $50-59(n=14)$ & $3(21.4)$ & $11(78.6)$ & \\
\hline $60-69(n=11)$ & $2(18.2)$ & $9(81.8)$ & \\
\hline $70-79(n=3)$ & $0(0)$ & $3(100.0)$ & \\
\hline Body mass index $\left(\mathrm{kg} / \mathrm{m}^{2}\right)$ & & & 0.499 \\
\hline < 18.5 (underweight, $n=2$ ) & $0(0)$ & $2(100.0)$ & \\
\hline 18.5-25.0 (normal weight, $\mathrm{n}=34$ ) & $5(14.7)$ & $29(85.3)$ & \\
\hline 25.1-30.0 (overweight, $n=15$ ) & $2(13.3)$ & $13(86.7)$ & \\
\hline$\geq 30.1$ (obese, $n=9$ ) & $3(33.3)$ & $6(66.7)$ & \\
\hline Presence of diabetes mellitus & & & 0.670 \\
\hline Yes $(n=7)$ & $1(14.3)$ & $6(85.7)$ & \\
\hline No $(n=53)$ & $9(17.0)$ & $44(83.0)$ & \\
\hline
\end{tabular}

Among the 60 patients, 10 (16.7\%) developed postoperative wound infections. Table I summarises the association between postoperative wound infections and the patient demographics. There was no statistical difference in the infection rates among patients from the different age groups or among patients of different genders. Patients with diabetes mellitus were found to be just as likely to develop a wound infection as a patient without diabetes mellitus. There was also no association between patient $\mathrm{BMI}$ and the rate of infection.

The demographic and surgical details of the patients who developed postoperative wound infections are summarised in Table II. Among these ten patients, seven developed minor superficial infections that were treated with antibiotics alone, while three developed deep wound infections. Among the three patients who had deep wound infections, two had a stitch granuloma each, while one had a gaping wound. The patients with the stitch granulomas required two separate surgical debridements complemented with oral antibiotics, while the patient with the gaping wound required one wound debridement followed by a split skin graft at a later date.

Although the overwhelming majority of the patients in the present study received preoperative antibiotics, $16.7 \%$ of them developed a wound infection. Table III shows the surgical factors associated with postoperative wound infections. Only one patient did not receive antibiotics and did not develop a wound infection. There were no reports of sural nerve injury or scar sensitivity. Only $6(10.0 \%)$ of the 60 patients smoked and only $1(16.7 \%)$ of these six patients had a wound infection. The patient with a wound infection was obese and also had diabetes mellitus. None 
Table II. Details of the patients with postoperative wound infections $(n=10)$.

\begin{tabular}{|c|c|}
\hline Demographic/surgical factor & No. (\%) \\
\hline \multicolumn{2}{|l|}{ Gender } \\
\hline Male & $5(50.0)$ \\
\hline Female & $5(50.0)$ \\
\hline \multicolumn{2}{|l|}{ Age group (yr) } \\
\hline $30-39$ & $3(30.0)$ \\
\hline $40-49$ & $2(20.0)$ \\
\hline $50-59$ & $3(30.0)$ \\
\hline $60-69$ & $2(20.0)$ \\
\hline \multicolumn{2}{|l|}{ Body mass index $\left(\mathrm{kg} / \mathrm{m}^{2}\right)$} \\
\hline < 18.5 (underweight) & $0(0)$ \\
\hline $18.5-25.0$ (normal weight) & $5(50.0)$ \\
\hline 25.1-30.0 (overweight) & $2(20.0)$ \\
\hline$\geq 30.1$ (obese) & $3(30.0)$ \\
\hline \multicolumn{2}{|l|}{ Presence of diabetes mellitus } \\
\hline Yes & $1(10.0)$ \\
\hline No & $9(90.0)$ \\
\hline \multicolumn{2}{|l|}{ Type of injury } \\
\hline Acute & $8(80.0)$ \\
\hline Chronic & $2(20.0)$ \\
\hline \multicolumn{2}{|l|}{ Surgical approach } \\
\hline Posteromedial & $10(100.0)$ \\
\hline Posterolateral & $0(0)$ \\
\hline \multicolumn{2}{|l|}{ Preoperative antibiotics } \\
\hline Yes & $10(100.0)$ \\
\hline No & $0(0)$ \\
\hline \multicolumn{2}{|l|}{ Postoperative antibiotics } \\
\hline Yes & $6(60.0)$ \\
\hline No & $4(40.0)$ \\
\hline \multicolumn{2}{|l|}{ Type of deep suture used } \\
\hline Ethibond & $3(30.0)$ \\
\hline Prolene & $4(40.0)$ \\
\hline Polydioxanone & $1(10.0)$ \\
\hline Vicryl & $1(10.0)$ \\
\hline Unknown & $1(10.0)$ \\
\hline \multicolumn{2}{|l|}{ Type of superficial suture used } \\
\hline Vicryl & $8(80.0)$ \\
\hline Others & $2(20.0)$ \\
\hline
\end{tabular}

of these six patients had major complications such as tendon re-ruptures, equinus deformity, DVT or PE at the follow-up two years after surgery.

\section{DISCUSSION}

Surgery on the foot is generally associated with a high rate of superficial wound infections $(8.2 \%-14.6 \%)$ and these infections are mostly related to surgeries on the TA. ${ }^{(4,5,7-9)}$ The vascular concept of angiosomes was first described in 1987.(10) Angiosomes are anatomic territories of skin, soft tissue and bone supplied by a single artery. Zones between angiosomes are formed by similar calibre or reduced calibre arteries that usually occur within the muscles rather than between them. ${ }^{(10-11)}$ Based on the theory of angiosomes, Attinger et al hypothesised
Table III. Surgical factors associated with wound infections.

\begin{tabular}{|c|c|c|c|}
\hline \multirow[t]{2}{*}{ Surgical factor } & \multicolumn{2}{|c|}{ No. (\%) } & \multirow[t]{2}{*}{ p-value } \\
\hline & $\begin{array}{c}\text { With } \\
\text { infection } \\
(n=10)\end{array}$ & $\begin{array}{c}\text { Without } \\
\text { infection } \\
(n=50)\end{array}$ & \\
\hline Type of injury & & & 0.400 \\
\hline Acute $(n=52)$ & $8(15.4)$ & $44(84.6)$ & \\
\hline Chronic $(n=8)$ & $2(25.0)$ & $6(75.0)$ & \\
\hline Surgical approach & & & 0.388 \\
\hline Posteromedial $(n=55)$ & $10(18.2)$ & $45(81.8)$ & \\
\hline Posterolateral $(n=5)$ & $0(0)$ & $5(100.0)$ & \\
\hline Preoperative antibiotics & & & 0.573 \\
\hline Yes $(n=59)$ & $10(16.9)$ & $49(83.1)$ & \\
\hline No $(n=1)$ & $0(0)$ & $1(100.0)$ & \\
\hline Postoperative antibiotics & & & 0.456 \\
\hline Yes $(n=32)$ & $6(18.8)$ & $26(81.3)$ & \\
\hline No $(n=28)$ & $4(14.3)$ & $24(85.7)$ & \\
\hline Type of deep suture used & & & 0.899 \\
\hline Ethibond $(n=20)$ & $3(15.0)$ & $17(85.0)$ & \\
\hline Prolene $(n=18)$ & $4(22.2)$ & $14(77.8)$ & \\
\hline Polydioxanone $(n=15)$ & $1(6.7)$ & $14(93.3)$ & \\
\hline Vicryl $(n=8)$ & $1(12.5)$ & $7(87.5)$ & \\
\hline Type of superficial suture used & & & 0.625 \\
\hline Vicryl $(n=38)$ & $8(21.1)$ & $30(78.9)$ & \\
\hline Prolene $(n=19)$ & 0 & $19(100.0)$ & \\
\hline Ethibond $(n=1)$ & 0 & $1(100.0)$ & \\
\hline Others $(n=2)$ & $2(100)$ & 0 & \\
\hline
\end{tabular}

that the safest incision for TA surgery would be a longitudinal midline incision on the TA itself, between the peroneal and posterior tibial artery angiosomes. ${ }^{(12)}$ If the skin incision was done either medially or laterally to the TA, it could disrupt the choke arteries and increase the risk of wound necrosis. The concept of the three zones of the hind foot, described by Yepes et al, contradicts the theory of angiosomes. ${ }^{(13)}$ In Yepes et al's study, the authors performed digital vascular mapping of five cadaveric TAs. They showed that there was rich blood supply medial to the TA, relatively good blood supply lateral to the TA and poor blood supply over the TA itself. ${ }^{(13)}$ The discrepancy in the theory of angiosomes and the concept of three hind foot zones highlights the need for further clinical-based research to elucidate the optimal placement of the skin incision during TA surgeries.

In the present study, we did not find any significant association between the gender of the patient and risk of infection (Table I). Although none of the patients aged between 20 and 29 years developed a wound infection, $16.7 \%-23.1 \%$ of the patients aged 30 to 69 years developed an infection. There was no difference in infection rates between normal-weight and overweight patients; however, patients who were obese were found to be two times more likely to develop an infection than patients of normal weight. There was no significant difference between the number of patients with diabetes mellitus who developed a wound infection and the number of patients without diabetes mellitus who developed a wound infection. However, it should be noted 
that the glycaemic control of the patients with diabetes mellitus was not analysed in the present study.

All but one of the patients in the present study received preoperative antibiotics (56 patients received cefazolin and three patients received vancomycin), yet 10 (16.7\%) patients still developed an infection. In a retrospective study performed by Zgonis et al, which included 555 patients who underwent elective foot and ankle surgeries, the rate of infection among the 306 patients who received preoperative antibiotics and 249 patients who did not was $1.6 \%$ and $1.4 \%$, respectively. ${ }^{(14)}$ The results of that study also showed that the rate of infection was not associated with age, gender, type of procedure, operative time, tourniquet use and past medical history. ${ }^{(14)}$ Although the study showed that the use of prophylactic antibiotics in elective foot and ankle surgery is not warranted, many surgeons continue to utilise it. In the Department of Orthopaedic Surgery at the Singapore General Hospital, perioperative intravenous cefazolin antibiotic prophylaxis is the default choice of antibiotics for orthopaedic procedures; intravenous vancomycin is used in patients who are allergic to penicillin or cephalosporins. Cefazolin is the most studied antimicrobial agent, with proven efficacy for antimicrobial prophylaxis. ${ }^{(15)}$ It has a desirable duration of action and spectrum of activity against organisms commonly encountered in surgery (i.e. it is active against streptococci, methicillin-susceptible staphylococci and some gram-negative organisms). It is also reasonably safe and inexpensive. Patients with penicillin allergies that manifest as an uncomplicated skin rash may still be treated with a cephalosporin as allergic cross-reactions between penicillin and cephalosporins are infrequent, except in patients with severe immunoglobulin E-mediated reactions to penicillin. Alternatives to cephalosporins include intravenous vancomycin and clindamycin. Although there is no role for the routine use of vancomycin prophylaxis in any procedure, its use in patients who have penicillin allergies and known colonisation with methicillinresistant Staphylococcus aureus (MRSA) or patients who have penicillin allergies and are at a high risk of MRSA colonisation (e.g. haemodialysis patients, nursing home residents or the recently hospitalised) is acceptable. ${ }^{(16,17)}$ Vancomycin may not be as effective as cefazolin in preventing surgical site infections caused by methicillin-susceptible $S$. aureus (MSSA), but due to the levels of community-acquired MRSA, surgeons may be inclined to use vancomycin as compared to clindamycin in patients who have penicillin allergies. ${ }^{(18-20)}$

In the present study, polydioxanone sutures $(6.7 \%)$ and Ethibond (15\%) sutures appeared to lead to a lower risk of infection when used to repair the ruptured TA (deep sutures), as compared to Ethibond (15\%), Prolene (22\%) and Vicryl (12.5\%) sutures. The difference, however, was not statistically significant. This may be due to the small study population. Vicryl sutures were used for skin closure for $80 \%$ of the infected wounds; none of the cases in which Prolene and Ethibond sutures were used for skin closure developed a wound infection. Once again, as the numbers involved were small, accurate conclusions may not be drawn from this result. However, it is still meaningful to compare the effects of the type of suture used when other factors such as patient age, patient gender and the presence of diabetes mellitus are matched. The type of suture used for skin closure has been shown to be closely correlated to the rate of surgical site infection. ${ }^{(21)}$ Prolene is a non-absorbable synthetic monofilament suture, while Ethibond is a non-absorbable synthetic braided suture. Different sutures of varying properties will have different effects on the surrounding tissue, and ultimately, the rate of wound infection. The monofilament nature of Prolene sutures elicits minimal inflammatory reaction in the surrounding tissue; the sutures are gradually encapsulated by fibrous connective tissue. Studies have also shown that Prolene sutures resist involvement in infected wounds. ${ }^{(22-24)}$ These studies showed that monofilament suture materials withstood contamination and infection considerably better than braided suture materials. The ability of sutures to resist infection is multifactorial. Bioadherence of bacteria to sutures is one important factor; monofilament polypropylene is less adherent than braided Ethibond. Furthermore, bacteria that reaches the interstices of the threads in braided sutures are protected from phagocytosis by leukocytes as there is no significant leukocyte penetration into the multifilament braided sutures. ${ }^{(25,26)}$ Recent studies have shown that surgical site infections can be reduced through the use of sutures that have been impregnated with antibiotics. ${ }^{(27-30)}$

The present study was not without limitations. Due to the fact that it was a retrospective study, it may have been subject to selection bias. Compromised patients who were deemed to be at a higher risk of infection due to age, poorly-controlled diabetes, long-term steroid use or significant smoking history may have been treated conservatively for these reasons. If this subset of patients had been treated surgically and included in the present study, the results may have been significantly different. The sample size of the present study and number of patients who developed wound infections were also small. It was therefore difficult to draw accurate conclusions or conduct any meaningful statistical test of significant power. We were not able to perform the GEE as there were an insufficient number of events (i.e. wound infections) among the small number of patients.

The patients included in the present study had their surgeries performed by ten different surgeons - either orthopaedic consultants or registrars. As orthopaedic consultants and orthopaedic registrars have different skill levels and experience, we acknowledge that a degree of bias is present in terms of patient selection, surgical approach, surgical time, repair technique and soft tissue handling; all these factors could have played a role in the risk of developing a wound infection after surgery. However, large orthopaedic units typically use a range of surgeons. Patients may be admitted as emergency or semi-emergency cases, and may be operated on by an orthopaedic surgeon in training or orthopaedic consultant. Furthermore, there is no standard protocol in our department regarding the way a TA repair should be done. Thus, the present study can be said to report the incidence of postoperative wound infection after TA open repairs, given the procedures in our department. The information obtained from the present study will enable our department, as well as others, to relook current practices. It also highlights the need for 
more robust protocols and research to analyse the efficacy of the new protocols in reducing infection rates.

There is considerable variation in functional bracing protocols in the literature. Some prefer cast immobilisation, while others promote functional rehabilitation in which weight-bearing mobilisation, with an early but limited range of movement, is permitted. ${ }^{(31-33)}$ Patients who undergo functional rehabilitation are given either a rigid rocker bottom orthosis or a more flexible, carbon-fibre dorsal orthosis. There is also debate surrounding the degree of plantar flexion the patients' ankles should be kept at. Some studies have advocated a plantigrade or neutral position with restricted dorsiflexion, while others are in favour of keeping the ankle in maximum plantar flexion to reduce the strain on the TA repair site. ${ }^{(34-36)}$ The American Academy of Orthopaedic Surgeons published its first clinical guidelines on the treatment of TA rupture in 2010. ${ }^{(37)}$ The authors recognised that despite the range of protocols and 'protective devices' available, there was a lack of clinical consensus regarding which devices should be used, the degree of ankle plantar flexion these devices should facilitate and how long these devices should be worn. ${ }^{(37)}$ It is clear from the report that there is increasing evidence to support the benefits of early functional rehabilitation. While we do not have a clear protocol in our department, the commonly chosen degree of plantar flexion is about $30^{\circ}$, to reduce strain over the repair site and also prevent excessive contracture of the TA. Lastly, in the present study, we did not analyse the glycaemic control of the patients with diabetes mellitus nor measure the strength of leg pulses in our patients. Future studies should consider looking into how these factors affect the risk of wound infection after open TA repair.

To conclude, in the present study, diabetes mellitus and age did not appear to be risk factors for postoperative wound infections. On the other hand, obese patients were found to be two times more likely to develop a wound infection than normalweight patients. The incidence of superficial wound infections in the present study was similar to previously published results $(11.7 \%$ vs. $8.2 \%-14.6 \%)$, but the incidence of deep infections was higher $(5 \%$ vs. $1 \%-2 \%) .{ }^{(4,5,7-9)}$

\section{REFERENCES}

1. Zhao HM, Yu GR, Yang YF, Zhou JQ, Aubeeluck A. Outcomes and complications of operative versus non-operative treatment of acute Achilles tendon rupture: a meta-analysis. Chin Med J (Engl) 2011; 124:4050-5.

2. Thevendran G, Sarraf KM, Patel NK, Sadri A, Rosenfeld P. The ruptured Achilles tendon: a current overview from biology of rupture to treatment. Musculoskelet Surg 2013; 97:9-20.

3. Tallon C, Maffuli N, Ewen SW. Ruptured Achilles tendons are significantly more degenerated than tendinopathic tendons. Med Sci Sports Exerc 2001; 33:1983-90.

4. Khan RJ, Fick D, Keogh A, et al. Treatment of acute achilles tendon ruptures. A meta-analysis of randomized, controlled trials. J Bone Joint Surg Am 2005; 87:2202-10.

5. Wong J, Barrass V, Maffulli N. Quantitative review of operative and nonoperative management of achilles tendon ruptures. Am J Sports Med 2002; 30:565-75.

6. van der Linden PD, Sturkenboom MC, Herings RM, et al. Increased risk of achilles tendon rupture with quinolone antibacterial use, especially in elderly patients taking oral corticosteroids. Arch Intern Med 2003; 163:1801-7.

7. Nilsson-Helander K, Silbernagel KG, Thomeé R, et al. Acute achilles tendon rupture: a randomized, controlled study comparing surgical and nonsurgical treatments using validated outcome measures. Am J Sports Med 2010; 38:2186-93.

8. Pajala A, Kangas J, Ohtonen P, Leppilahti J. Rerupture and deep infection following treatment of total Achilles tendon rupture. J Bone Joint Surg Am 2002; 84-A:2016-21.

9. Highlander P, Greenhagen RM. Wound complications with posterior midline and posterior medial leg incisions: a systematic review. Foot Ankle Spec 2011; 4:361-9.

10. Taylor GI, Palmer JH. The vascular territories (angiosomes) of the body: experimental study and clinical applications. Br J Plast Surg 1987; 40:113-41.

11. Taylor GI, Pan WR. Angiosomes of the leg: anatomic study and clinical implications. Plast Reconstr Surg 1998; 102:599-616.

12. Attinger CE, Evans KK, Bulan E, Blume $P$, Cooper P. Angiosomes of the foot and ankle and clinical implications for limb salvage: reconstruction, incisions, and revascularization. Plast Reconstr Surg 2006; 117(7 Suppl):261S-293S

13. Yepes $H$, Tang $M$, Geddes $C$, et al. Digital vascular mapping of the integument about the Achilles tendon. J Bone Joint Surg Am 2010; 92:1215-20.

14. Zgonis T, Jolly GP, Garbalosa JC. The efficacy of prophylactic intravenous antibiotics in elective foot and ankle surgery. J Foot Ankle Surg 2004; 43:97-103.

15. Bratzler DW, Dellinger EP, Olsen KM, et al; American Society of HealthSystem Pharmacists (ASHP); Infectious Diseases Society of America (IDSA); Surgical Infection Society (SIS); Society for Healthcare Epidemiology of America (SHEA). Clinical practice guidelines for antimicrobial prophylaxis in surgery. Surg Infect (Larchmt) 2013; 14:73-156.

16. Anderson DJ, Podgorny K, Berríos-Torres SI, et al. Strategies to prevent surgical site infections in acute care hospitals: 2014 update. Infect Control Hosp Epidemiol 2014; 35:605-27.

17. Gupta K, Strymish J, Abi-Haidar Y, Williams SA, Itani KM. Preoperative nasal methicillin-resistant Staphylococcus aureus status, surgical prophylaxis, and risk-adjusted postoperative outcomes in veterans. Infect Control Hosp Epidemiol 2011; 32:791-6.

18. Finkelstein R, Rabino G, Mashiah T, et al. Vancomycin versus cefazolin prophylaxis for cardiac surgery in the setting of a high prevalence of methicillin-resistant staphylococcal infections. J Thorac Cardiovasc Surg 2002; 123:326-32.

19. Bull AL, Worth LJ, Richards MJ. Impact of vancomycin surgical antibiotic prophylaxis on the development of methicillin-sensitive staphylococcus aureus surgical site infections: report from Australian Surveillance Data (VICNISS). Ann Surg 2012; 256:1089-92.

20. Kanj WW, Flynn JM, Spiegel DA, Dormans JP, Baldwin KD. Vancomycin prophylaxis of surgical site infection in clean orthopedic surgery. Orthopedics 2013; 36:138-46.

21. Alexander JW, Solomkin JS, Edwards MJ. Updated recommendations for control of surgical site infections. Ann Surg 2011; 253:1082-93.

22. Alexander JW, Kaplan JZ, Altemeier WA. Role of suture materials in the development of wound infection. Ann Surg 1967; 165:192-9.

23. Varma S, Ferguson HL, Breen H, Lumb WV. Comparison of seven suture materials in infected wounds--an experimental study. J Surg Res 1974; 17:165-70.

24. Sharp WV, Belden TA, King PH, Teague PC. Suture resistance to infection. Surgery 1982; 91:61-3.

25. Chu CC, Williams DF. Effects of physical configuration and chemical structure of suture materials on bacterial adhesion. A possible link to wound infection. Am J Surg 1984; 147:197-204.

26. Osterberg B. Enclosure of bacteria within capillary multifilament sutures as protection against leukocytes. Acta Chir Scand 1983; 149:663-8.

27. Gilbert P, McBain AJ. Literature-based evaluation of the potential risks associated with impregnation of medical devices and implants with triclosan. Surg Infect (Larchmt) 2002; 3 Suppl 1:S55-63.

28. Edmiston CE, Seabrook GR, Goheen MP, et al. Bacterial adherence to surgical sutures: can antibacterial-coated sutures reduce the risk of microbial contamination? J Am Coll Surg 2006; 203:481-9.

29. Ming $X$, Nichols $M$, Rothenburger S. In vivo antibacterial efficacy of MONOCRYL plus antibacterial suture (Poliglecaprone 25 with triclosan). Surg Infect (Larchmt) 2007; 8:209-14.

30. Ming X, Rothenburger S, Nichols MM. In vivo and in vitro antibacterial efficacy of PDS plus (polidioxanone with triclosan) suture. Surg Infect (Larchmt) 2008; 9:451-7.

31. Wallace RG, Heyes GJ, Michael AL. The non-operative functional management of patients with a rupture of the tendo Achillis leads to low rates of re-rupture. J Bone Joint Surg Br 2011; 93:1362-6. 
32. Garrick JG. Does accelerated functional rehabilitation after surgery improve outcomes in patients with acute achilles tendon ruptures? Clin J Sport Med 2012; 22:379-80.

33. Olsson N, Silbernagel KG, Eriksson Bl, et al. Stable surgical repair with accelerated rehabilitation versus nonsurgical treatment for acute Achilles tendon ruptures: a randomized controlled study. Am J Sports Med 2013 41:2867-76.

34. Speck M, Klaue K. Early full weightbearing and functional treatment after surgical repair of acute achilles tendon rupture. Am J Sports Med 1998; 26:789-93
35. Costa ML, MacMillan K, Halliday D, et al. Randomised controlled trials of immediate weight-bearing mobilisation for rupture of the tendo Achillis. J Bone Joint Surg Br 2006; 88:69-77.

36. Bhattacharyya M, Gerber B. Mini-invasive surgical repair of the Achilles tendon--does it reduce post-operative morbidity? Int Orthop 2009; 33:151-6.

37. Chiodo CP, Glazebrook M, Bluman EM, et al; American Academy of Orthopaedic Surgeons. American Academy of Orthopaedic Surgeons clinical practice guideline on treatment of Achilles tendon rupture. J Bone Joint Surg Am 2010; 92:2466-8. 